\title{
Cerebellar nucleus lesions dissociate alpha conditioning from alpha responses in rabbits
}

\author{
RONALD W. SKELTON, MICHAEL D. MAUK, and RICHARD F. THOMPSON \\ Stanford University, Stanford, California
}

\begin{abstract}
This experiment compared the effects of unilateral lesions of the cerebellar nuclei and control lesions of the cerebellar cortex on alpha responses, alpha conditioning, and classical conditioning in the rabbit eyelid conditioning paradigm. Lesions of the dentate-interpositus (DI) region of the cerebellar nuclei abolished retention of conditioned responses to an auditory conditioned stimulus (CS), and prevented acquisition of conditioned alpha responses to a dim proximal light CS, but did not diminish alpha responses to a bright proximal light. These results indicate that the DI region is essential to both alpha conditioning and classical conditioning of eyelid responses, but not to alpha responses, and suggest that both forms of conditioning are mediated by a common anatomical substrate that is distinct from the anatomical substrate of alpha responses.
\end{abstract}

Recent neurobiological investigations of learning and memory have emphasized the benefits of using either simple nervous systems or reduced preparations, and have made substantial progress in understanding the cellular basis of habituation, sensitization, and classical conditioning (see Byrne, 1987). A fundamental principle of this approach is that complex behaviors and nervous systems both evolved from simpler ones, and that cellular processes of learning may have been preserved across phylogeny. However, one issue of continuing controversy is the degree to which findings in these model systems generalize to learning by intact vertebrates, and a major point of contention is the use of a somewhat atypical form of learning called alpha conditioning.

Alpha conditioning is the maintenance or augmentation of an unconditioned, or alpha, response to the conditioned stimulus (CS) through classical conditioning procedures (Gormezano \& Moore, 1969; Kandel \& Spencer, 1968). Historically, alpha conditioning was considered to be quite distinct from beta conditioning, which produced a conditioned response (CR) functionally similar to the unconditioned response (UR) elicited by the unconditioned stimulus (US) (Hull, 1934). Currently, alpha conditioning is still considered to be distinct from beta conditioning (now called simply classical conditioning; Gormezano \& Moore, 1969), because the former involves the intensification of a preexisting response to the CS, whereas

This research was funded by Grant BNS-8117115 from the National Science Foundation and Grant N00014-83 from the Office of Naval Research to R. F. Thompson. We gratefully acknowledge the assistance of those who provided helpful comments during the writing of this manuscript: A. Black, G. Clark, M. Corcoran, R. Dixon, and N. Donegan. Please address all correspondence to R. W. Skelton, Department of Psychology, University of Victoria, Box 1700, Victoria, British Columbia V8W 2Y2, Canada. Michael D. Mauk is currently at the Department of Neurobiology and Anatomy, University of Texas Medical School, Houston, TX 77225. Richard F. Thompson is currently at the Department of Psychology, University of Southern California, University Park, Los Angeles, CA 90089-1061. the latter requires the development of a new CR to the CS (Gormezano \& Kehoe, 1975). The concern with the use of behavioral models based on alpha conditioning is that alpha conditioning and classical conditioning may be mediated by fundamentally different mechanisms. After all, alpha conditioning could be accomplished simply by enhancing the prepotent alpha response pathway, whereas classical conditioning presumably requires a new pathway for the $\mathrm{CR}$.

Conversely, it could be argued that at the neural level, alpha conditioning and classical conditioning are the same. Neurobiologists generally agree that most, if not all, learning is mediated by changes in preexisting neural circuits, rather than the formation of new ones (Byrne, 1987). If both alpha and classical conditioning depend on changes in preexisting circuits, then the only difference between them is in the strength of these circuits prior to learning. The logical extensions of this argument, made by Carew, Abrams, Hawkins, and Kandel (1984), are, first, that behavioral alpha responses should be observable in almost any classical conditioning paradigm, simply by increasing the intensity of the CS, and, second, that neuronal alpha responses should be measurable as increased excitability levels of neurons in premotor structures. Indeed, Carew et al. (1984) maintained that all classical conditioning could be mediated by augmentation of a prepotent, or nearly prepotent, alpha response pathway, as it is in the Aplysia.

Although these arguments are compelling, the actual relationship between alpha conditioning and classical conditioning in mammals remains unknown. Presumably, the two forms of conditioning could be quite distinct, and mediated by entirely different mechanisms. Alternatively, they could differ only in the magnitude of the preexisting response to the CS, and could be mediated by a common neuronal mechanism; this common mechanism might or might not involve changes in a prepotent, or nearly prepotent, alpha response pathway. 
Direct comparison of the neural bases of alpha and classical conditioning is now possible, because of two important features of eyelid conditioning in the rabbit. First, both forms of conditioning can be obtained in this paradigm. Although alpha responses are not elicited by auditory or diffuse visual CSs (Gormezano, 1972; Patterson \& Romano, 1987), alpha responses and alpha conditioning can be obtained if the CS is a localized light, held proximal to the eye being tested (an incidental observation by Skelton, Donegan, \& Thompson, 1984). As illustrated in Figure 1, there is a remarkable similarity among the response topographies of a CR to a tone, a conditioned alpha response to a dim proximal light, and an alpha response to a bright proximal light.

The second important feature of rabbit eyelid conditioning is that a substantial portion of the pathway mediating CRs has been identified, and it is possible to selectively disrupt eyelid CRs without affecting URs to the US. Briefly, known portions of this pathway include mossy fiber inputs to the cerebellum, the cerebellar deep nuclei, projections of the deep nuclei to the contralateral red nucleus via the superior cerebellar peduncle, the red

\section{ALPHA RESPONSE}

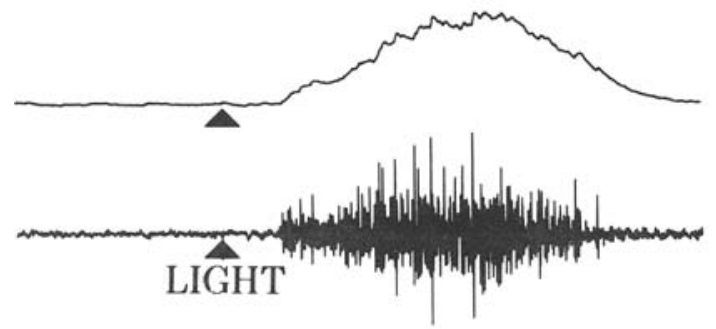

nucleus, and descending rubrobulbar projections to the motor nuclei of the extraocular and eyelid muscles (see Thompson, 1986, for a more complete description). Lesions at any point along this pathway completely and permanently abolish eyelid CRs, without diminishing URs to a corneal airpuff or extraocular electric shock (McCormick, Clark, Lavond, \& Thompson, 1982; McCormick \& Thompson, 1984; Rosenfield, Devydaitis, \& Moore, 1985; Solomon, Lewis, LoTurco, Steinmetz, \& Thompson, 1986; Yeo, Hardiman, \& Glickstein, 1985). Although the exact locus and nature of the neuronal plasticity subserving the $C R$ remain unidentified, the dentateinterpositus (DI) region of the cerebellar nuclei is clearly essential: Small lesions of the DI abolish CRs to visual, auditory, and vibratory stimuli only in the ipsilateral eye (McCormick \& Thompson, 1984; Yeo et al., 1985). In contrast, substantial lesions of the overlying ansiform cerebellar cortex have no lasting effect (McCormick \& Thompson, 1984).

The objective of the present experiment was to determine whether alpha responses and alpha conditioning depend upon the same anatomical structures that subserve

\section{CONDITIONED RESPONSES}
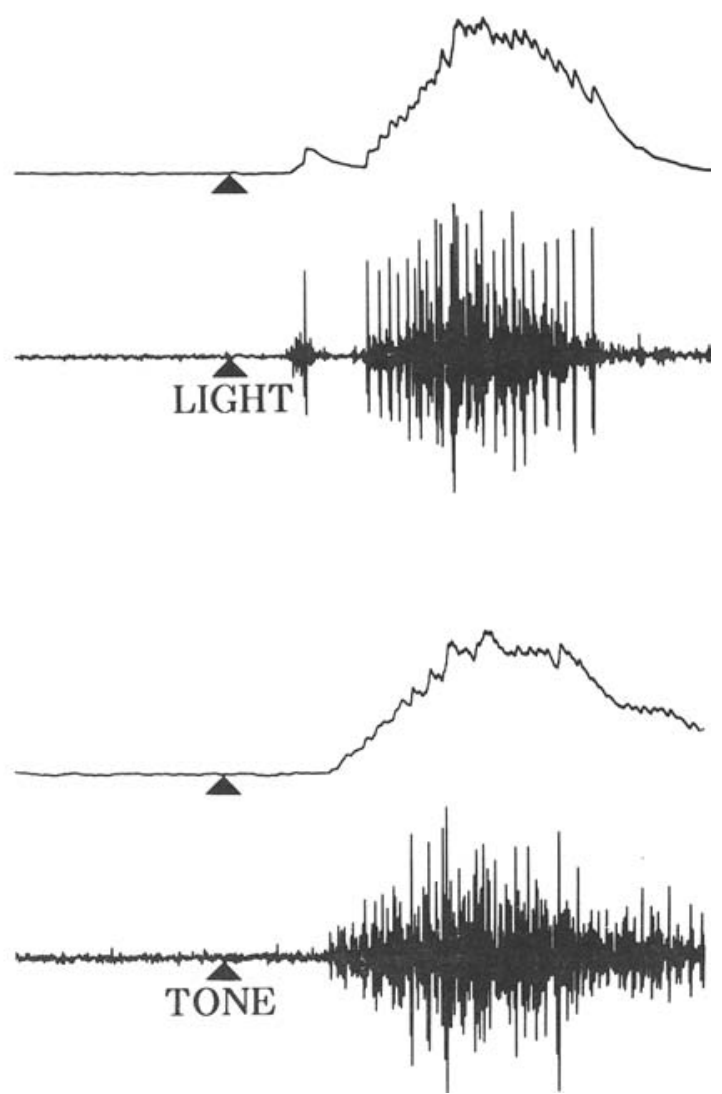

Figure 1. Individual eyelid responses to three different stimuli by a single rabbit illustrate the topographic similarity of alpha responses to bright lights, conditioned responses to dim lights, and conditioned responses to tones. Lower traces are raw electromyograms, upper traces are the integrated form of the same responses. Triangles denote the onset of each stimulus (no airpufis were administered on these trials); calibrations are $0.1 \mathrm{mV}$ and $250 \mathrm{msec}$. 
classical conditioning of eyelid CRs. Lesions of the DI region were used to disrupt the CR pathway, and the effects of this damage on alpha responses, alpha conditioning, and classical conditioning were evaluated. Logically, deficits in all three would indicate that all three depend on the same anatomical structures and would support the argument that classical conditioning involves augmentation of a prepotent or nearly prepotent pathway. Alternatively, deficits in only classical conditioning would indicate that alpha and classical conditioning of eyelid responses have different anatomical substrates, and would support the arguments that they are distinct forms of conditioning, and that alpha conditioning involves augmentation of the alpha response pathway. Finally, deficits in both alpha and classical conditioning, with no deficits in alpha responses, would indicate that both forms of eyelid conditioning depend on adjoining, if not identical, anatomical structures, and that neither form of conditioning depends on changes in the prepotent alpha response pathway.

\section{METHOD}

Briefly, this experiment compared the effects of cerebellar nucleus lesions and cerebellar cortical lesions on alpha responses to a bright light, conditioned alpha responses to a dim light, and CRs to a tone. Eyelid responses were recorded as electromyographic (EMG) activity. First, alpha responses to a bright light were measured bilaterally and CRs to a tone CS were trained unilaterally over two daily sessions in a Pavlovian delay paradigm. Then unilateral electrolytic lesions of the cerebellar cortex or DI region were made through chronically implanted electrodes and the rabbits were allowed to recover. Next, all rabbits were tested for preservation of alpha responses to the bright light and CRs to the tone. Alpha responses contralateral to the lesions were used to control for nonassociative changes and nonspecific effects of the lesions. Finally, acquisition of CRs to a dim light CS was evaluated for rabbits in both lesion groups. Half of the rabbits in each lesion group were given unpaired rather than paired presentations of the dim-light CS and airpuff US, in order to assess nonassociative changes in responding. No attempt was made to train CRs to the dim light prior to the lesions, in order to avoid confounding learned and unlearned responses to visual stimuli.

\section{Subjects}

Sixteen adult male New Zealand White rabbits (Oryctolagus cuniculus) weighing $2-3 \mathrm{~kg}$ were housed individually, given food and water ad lib, and maintained on a 12:12-h light:dark cycle. All testing took place during the light portion of the cycle.

\section{Surgery}

Surgery was conducted under Halothane gas anesthesia (2\%-5\% in $\mathrm{O}_{2}$ ), using a custom stereotaxic instrument that held the surface of the skull level (i.e., bregma was $1.5 \mathrm{~mm}$ dorsal to lambda). Bilateral EMG recording electrodes were made by passing stainless steel wire through the skin of the upper eyelid over the orbicularis oculi muscles of each eye and attaching the ends of the resulting loop to a connector pin (Amphenol). In the experimental group $(n=8)$, the intracerebellar lesion electrodes (No. 00 stainless steel insect pins insulated to within $0.5 \mathrm{~mm}$ of the tip) were implanted into the left DI region: $0.5 \mathrm{~mm}$ posterior, $6.5 \mathrm{~mm}$ lateral, and $13.0 \mathrm{~mm}$ ventral to lambda. In the control group $(n=8)$, the electrodes were implanted into the cerebellar cortex, $3.0 \mathrm{~mm}$ dor- sal to the DI, using the same lateral and posterior coordinates. The lesion electrode, plus a head stage for attaching recording leads and brackets for lights and airpuff nozzle, were cemented in place with dental acrylic and affixed to the skull with stainless steel screws. The rabbits were given 3-5 days to recover from surgery.

\section{Apparatus}

Behavioral tests took place inside a dimly lit sound-attenuating chamber, with the rabbits restrained in Plexiglas boxes. Recording leads, lights, and the airpuff nozzle were attached to the head stage. The lights for presenting visual stimuli were 6-V flashlight bulbs suspended $1 \mathrm{~cm}$ in front of each eye. Bright light $\left(3,000 \mathrm{~lm} / \mathrm{m}^{2}\right.$ at $\left.1 \mathrm{~cm}\right)$ was presented by driving a bulb with $6.5 \mathrm{~V} \mathrm{dc}$; dim light $\left(375 \mathrm{~lm} / \mathrm{m}^{2}\right.$ at $1 \mathrm{~cm}$ ) was presented by driving a bulb with $3 \mathrm{~V}$ dc. During the intertrial interval (ITI), the filament of the bulb being tested was preheated to a red glow in order to reduce the CS onset latency to less than $30 \mathrm{msec}$. The tone CS $(3 \mathrm{kHz}, 85 \mathrm{~dB}$ SPL at $15 \mathrm{~cm}$ ) was presented through a speaker mounted $15 \mathrm{~cm}$ in front of the rabbit's ears. On any given trial, the $350-$ msec CS was a bright light, a dim light, or a tone. The 100 -msec US (if present) was an airpuff $\left(2.1 \mathrm{~N} / \mathrm{cm}^{2}\right.$ at the source) delivered to the cornea of the left eye through a nozzle mounted on the same bracket as the light bulb. On CS-US paired trials, the CS preceded the US by $250 \mathrm{msec}$ and the CS and US coterminated. A 250-msec baseline period began each trial and preceded the CS. Presentation and timing of stimuli was controlled by a KIM microcomputer.

Eyelid EMG was amplified using a custom amplifier (Brakel, Babb, Mahnke, \& Verzeano, 1971; gain $=20,000$, bandpass $0.5-5 \mathrm{kHz}$ ), displayed on a Tektronix storage oscilloscope, and recorded on a four-channel audio tape recorder, along with synchronization pulses indicating the timing of trial periods. After the session, the recorded EMG was integrated using a custom integrator (45 msec time constant), and digitized ( $333 \mathrm{~Hz}$ ) by a DEC 11/03 minicomputer in synchrony with the trial-period markers. Eyelid CRs were measured during the first $250 \mathrm{msec}$ after CS onset (i.e., the CS-US interval when the US was present); URs were measured over the subsequent 250 -msec period; alpha responses were measured over both 250 -msec periods. Alpha responses, CRs, and URs were all measured for amplitude, onset latency, peak latency, and response area (amplitude $x$ time), and average response waveforms were calculated.

\section{Procedures}

Prelesion. After recovery from initial surgery, alpha responses to bright light were tested bilaterally, and CRs to tone were trained in the left eye over two daily test sessions. All sessions lasted $1 \mathrm{~h}$ and began with a 10-min habituation period to allow the rabbits to adapt to the restraining box, head stage, and low level of ambient illumination. Alpha responses were recorded in 4 blocks of nine CS-alone trials in which the bright-light CS was presented first to the left eye, then to the right. Then CRs to the tone were trained over 10 blocks of paired conditioning trials. Each block consisted of one CS-alone test trial plus eight conditioning trials in which the tone CS was paired with an airpuff US to the left eye. Trials were presented on a variable-interval (VI) 30 -sec intertrial interval.

Lesions. Unilateral electrolytic lesions were made under light Halothane anesthesia $\left(2 \%-5 \%\right.$ in $\left.\mathrm{O}_{2}\right)$ by passing $2.0 \mathrm{~mA}$ anodal direct current through the chronic lesion electrode. The rabbits were given 3-5 days to recover.

Postlesion. Alpha responses to the bright light and CRs to the tone were retested in a single session by repeating the prelesion schedule of trials: 2 blocks of bright-light CS presented to the left eye, 2 blocks of bright-light CS presented to the right eye, and 10 blocks of tone CS paired with airpuff US to the left eye. Then acquisition of alpha conditioning and pseudoconditioning to the dim- 
light CS were evaluated over four sessions. Four of the rabbits in each lesion group received paired presentations of the dim-light CS and airpuff US in 13 blocks of paired conditioning trials. Once again, each block contained eight CS-US conditioning trials plus one CSalone test trial. The remaining 4 rabbits in each lesion group received an equivalent number of unpaired presentations of the same CS and US, that is, 13 blocks of eight CS-alone plus eight US-alone trials presented in pseudorandom order on a VI 15-sec schedule.

Histology. After the conclusion of all testing, the rabbits were sacrificed with an overdose of Nembutal and perfused with saline followed by formalin. Lesion-induced damage to the cerebellum was determined using standard histological procedures for cell-body stains.

\section{RESULTS}

The effects of DI lesions on alpha responses, CRs, and conditioned alpha responses are described first in overview and then in detail.

Prior to the lesions, and indeed from the outset of testing, all rabbits displayed robust alpha responses to the bright light (Figure 2). Over the course of prelesion conditioning, all rabbits also acquired large and reliable CRs to the tone CS (Figure 2). In fact, by the end of the 2 prelesion test days, CRs and alpha responses had very similar amplitudes and topographies (see Figures 1 and 2 ). The behavioral similarity of alpha and conditioned responses suggests that the CRs could have developed through augmentation of the alpha response pathway. Therefore, this paradigm provided an excellent opportunity to test whether a common substrate subserved these two topographically indistinguishable responses.

Lesions of the DI abolished retention of CRs to the tone CS and blocked acquisition of conditioned alpha responses to the dim-light CS, but did not impair unconditioned al-

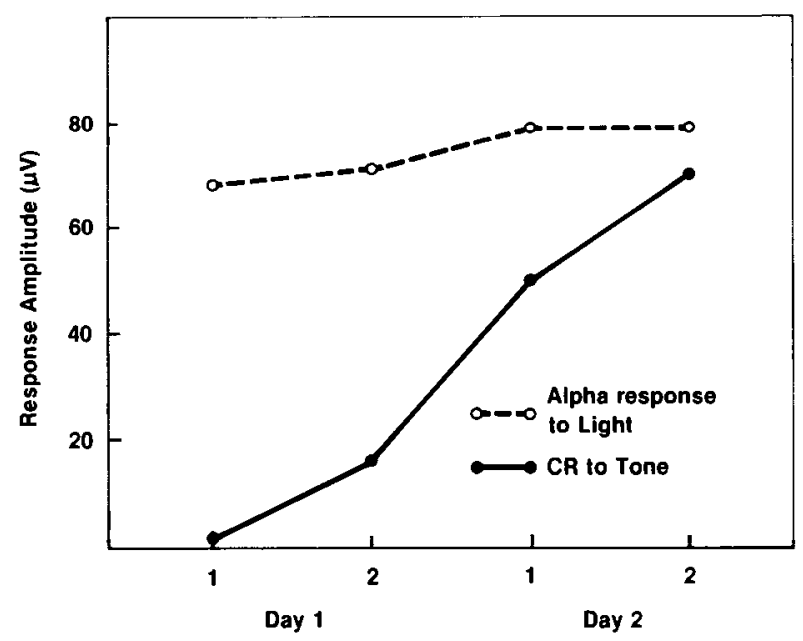

Figure 2. Prelesion amplitudes of alpha responses to bright light and conditioned responses (CR) to a tone are shown for the first and second halves of the 2 prelesion test days. Peak amplitudes of alpha responses were measured on each trial and averaged over both eyes of all 16 rabbits; peak amplitudes of CRs were measured only from the trained eye, on each paired trial, and were also averaged over all 16 rabbits. pha responses to the bright light. In contrast, lesions of the cerebellar cortex did not impair retention of CRs to the tone, did not block acquisition of conditioned alpha responses to the dim-light CS, and appeared to enhance alpha responses to the bright light. This pattern of deficits indicates that alpha conditioning in the present experiment was mediated by neural elements similar to those mediating classical conditioning, and that these elements were distinct from those mediating the alpha response.

\section{Prelesion}

Large alpha responses to the bright light were observed throughout the prelesion tests (see Figure 2), and tended to show sensitization rather than habituation. Mean alpha response amplitudes, averaged over both eyes of all 16 animals, rose $13 \%$ from the start of Day 1 to the end of Day $2(69.5 \mu \mathrm{V}$ to $79.1 \mu \mathrm{V})$. In contrast, CRs to the tone CS appeared only after training: mean CR amplitude increased almost 30 -fold, from $2.3 \mu \mathrm{V}$ on the first half of Day 1 to $70.4 \mu \mathrm{V}$ on the last half of Day 2. By the end of prelesion testing, mean amplitudes of CRs and alpha responses were remarkably similar $(M \mathrm{~s}=70.4$ and $79.1 \mu \mathrm{V}$, respectively).

\section{Lesion}

Lesions targeted to the DI region destroyed the dorsolateral portion of the interpositus nucleus and the dorsomedial portion of the dentate nucleus, as well as portions of the overlying ansiform cortex and hemispheral lobule VI (see Figure 3). Control lesions targeted to the cortex dorsal to the DI produced substantial damage to the same regions of the ansiform cortex and hemispheral lobule VI, and did not extend into the deep nuclei (see Figure 3).

\section{Postlesion}

Neither DI nor cortical lesions impaired alpha responses ipsilaterally or contralaterally (see Figure 4). Responses ipsilateral to the DI lesion were $118 \% \pm 10 \%$ of prelesion baseline amplitude, and were comparable in size to control responses contralateral to the DI lesion, which were $130 \% \pm 9 \%$ of prelesion baseline. These responses were similar not only to each other, and to prelesion responses, but also to responses contralateral to cortical lesions, which were $120 \% \pm 15 \%$ of prelesion baseline. Unexpectedly, responses ipsilateral to cortical lesions were much larger after the lesions than before $(176 \pm 38 \%)$, and were significantly different from responses in the contralateral eye and responses ipsilateral to DI lesions $[p<.05$, Tukey HSD pairwise comparisons, following a significant ANOVA, $F(3,28)=3.58, p<.05$ ]. No other pairs of means were significantly different. In sum, alpha responses contralateral to either lesion and ipsilateral to" the DI lesion were unaffected by the lesions, but alpha responses ipsilateral to the cortical lesion were significantly enhanced. This pattern of results suggests that cortical lesions specifically potentiated alpha responses in the ipsilateral eye and that DI lesions had no effect. 


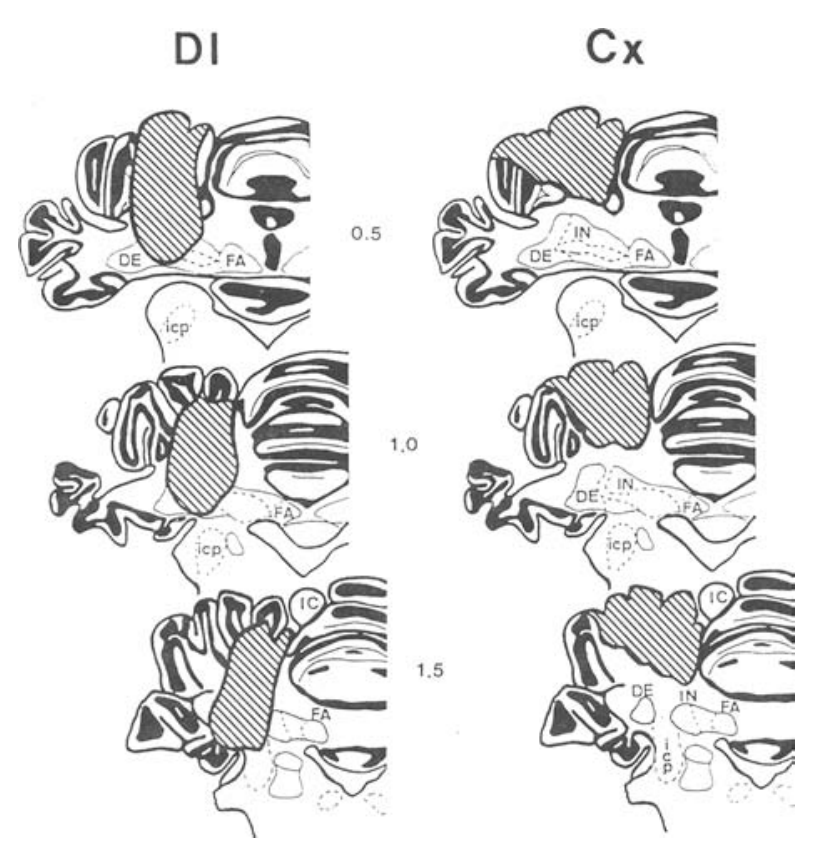

Figure 3. Damage due to lesions of the dentate-interpositus (DI) or cortex $(\mathbf{C x})$ of the cerebellum are depicted on drawings of partial coronal sections (adapted from McCormick \& Thompson, 1982). Striped areas represent the maximal extent (range) of all lesions within each group ( $n=8$ for cortical lesions, $n=4$ for DI lesions). Numbers indicate the rostral distance of each section from lambda in millimeters. DE = dentate nucleus, $I C=$ inferior colliculus, icp $=$ inferior cerebellar peduncle, $\mathbf{I N}=$ interpositus nucleus, $\mathbf{F A}=$ fastigial nucleus. (Accurate placement of lesions was confirmed in all rabbits, but lesion documentation for 4 DI-lesioned rabbits was subsequently lost.)
Lesions of the DI severely disrupted CRs to the tone CS, and cortical lesions had little or no effect (see Figure 4). Postlesion CR amplitudes in the DI lesion group were very small $(M=13 \% \pm 6 \%$ of prelesion levels), and were significantly different from CR amplitudes in the cortical lesion group $[M=128 \% \pm 19 \%, t(14)$ $=5.68, p<.001]$. These results were entirely consistent with previous reports (e.g., McCormick \& Thompson, 1984; Yeo et al., 1985), and provided strong behavioral evidence that both cortical and DI lesions were accurately placed.

Lesions of the DI did not eliminate alpha responses to the dim light but blocked both pseudoconditioning and alpha conditioning. Initially, all four groups of rabbits displayed small alpha responses to the dim light CS (see Figure 5). However, over the early training trials with the dim-light CS, response areas (amplitude $x$ duration) in the cortical lesion group increased dramatically, regardless of CS-US contiguity. Over the next 3 days of testing, responses in the cortical lesion group continued to increase, but only if the CS and US were paired. If the CS and US were unpaired, response areas stabilized and then declined. Response areas in the DI lesion group remained uniformly small from the beginning of testing to the end.

The initial and rapid increase in responding by cortically lesioned rabbits was clearly due to pseudoconditioning, because it did not depend upon contiguity between the CS and US. A three-way repeated measures analysis of variance (ANOVA) (lesion $\times$ contiguity $\times$ trials) on response areas for the first 16 trials revealed a significant interaction between lesions and trials $[F(7,84)=$

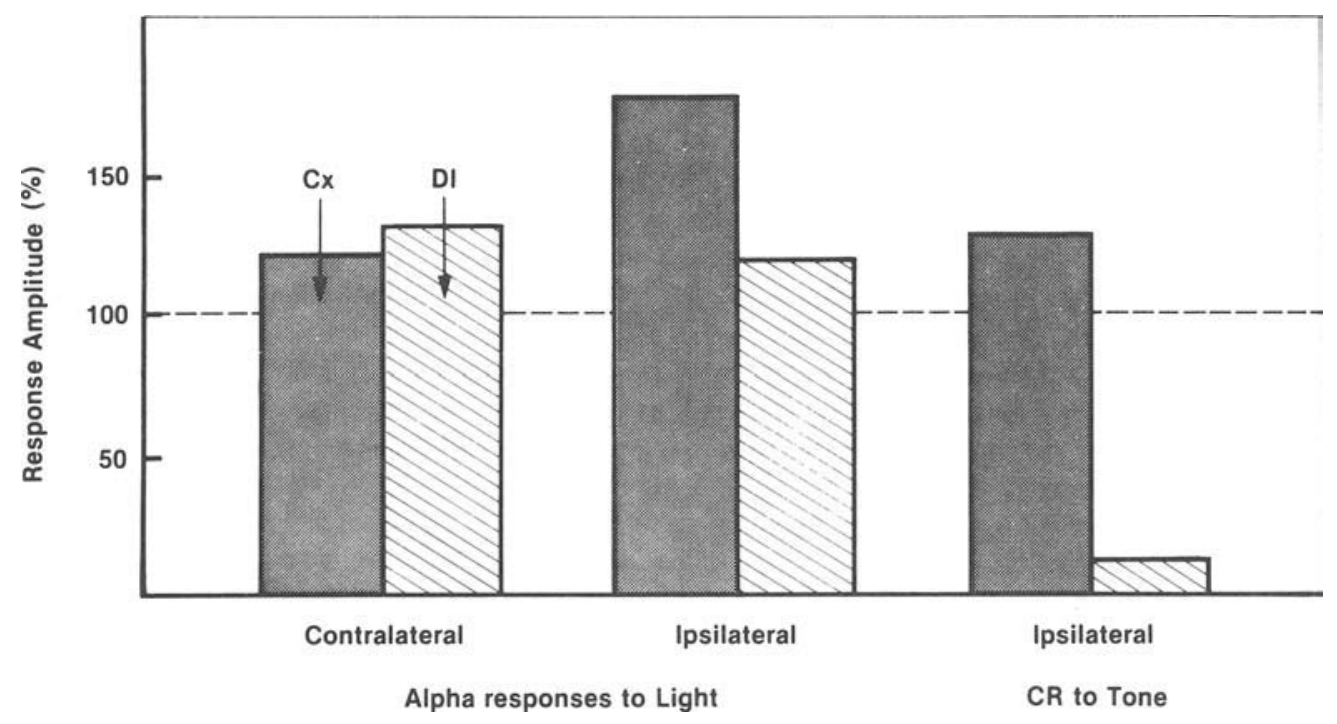

Figure 4. Changes in the amplitudes of alpha responses and conditioned responses (CR) as a function of lesion type (cortex, $\mathbf{C x}$, vs. dentate-interpositus, DI) and anatomical relationship between lesion and eye (ipsilateral vs. contralateral). Postlesion amplitudes are expressed as a percentage of prelesion levels, using both prelesion test days as baseline for alpha responses but only the last test day for CRs. 


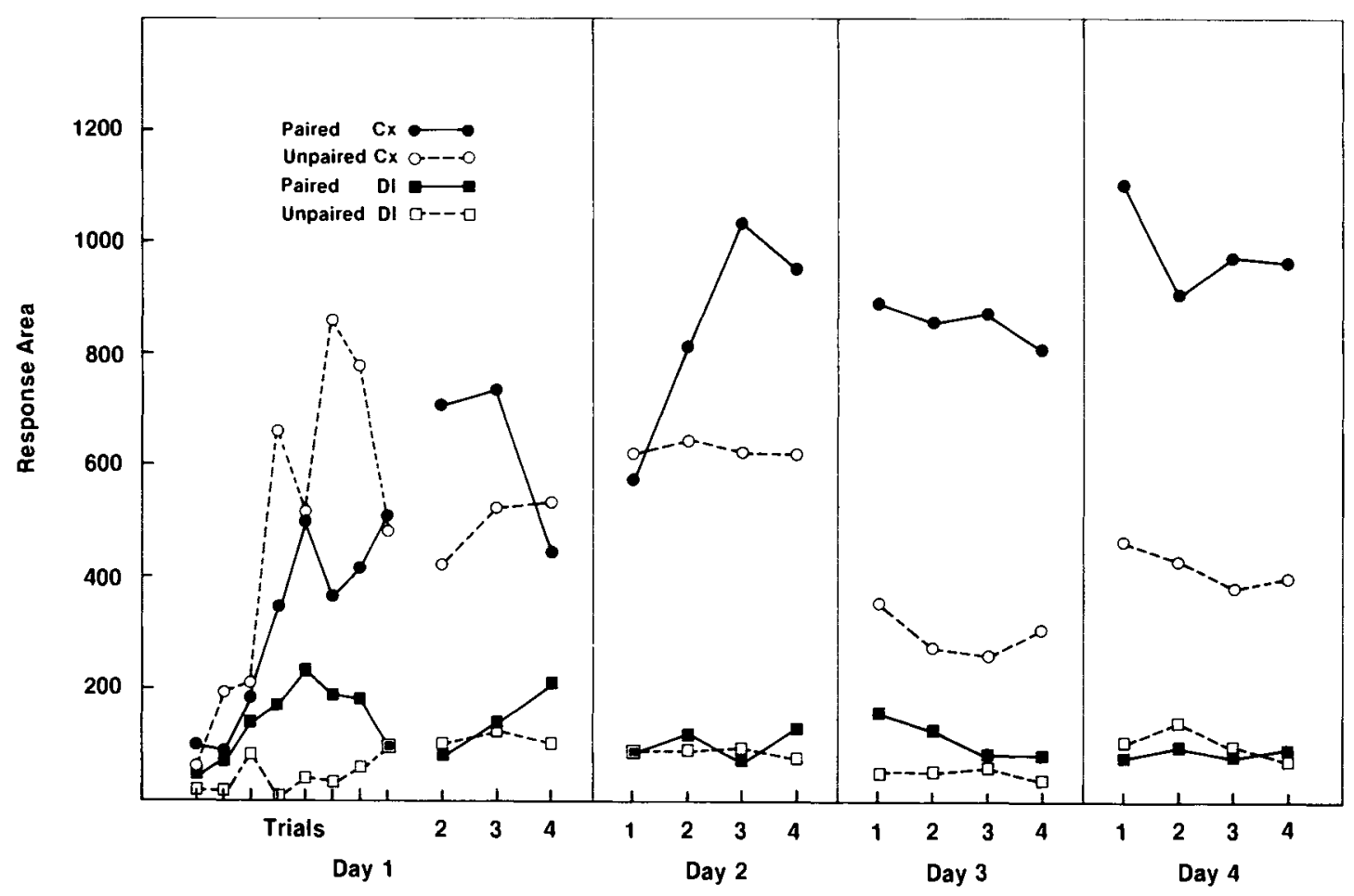

Figure 5. Postlesion conditioned responses to dim lights as a function of training (days), CS-US contiguity (Paired vs. Unpaired) and lesion type (cortex, Cx, vs. dentate-interpositus, DI). Response magnitudes are expressed in units of area (amplitude $\times$ time: $\mu V$-msec) measured during the CS-US interval of each trial and averaged within each combination of lesion and CS-US contiguity ( $n s=4$ in all cases). Amplitudes are shown averaged over each quarter of the 4 training days, except for the first two blocks of the first day, which are shown as average amplitudes of pairs of trials.

$2.38, p<.05$ ], confirming the observation that the changes in responses over trials were different for the two lesion groups. There was no effect of CS-US contiguity, either as a main effect $[F(1,12)=.01, n s$. $]$ or as an interaction with lesions $[F(1,12)=0.75, n s$.] or trials $[F(1,12)=0.63$, n.s. $]$ or with lesions and trials $[F(7,84)$ $=1.20$, n.s.]. In other words, both paired and unpaired CS-US presentations led to differential response increases in the cortical and DI lesion groups.

The most important observation in this experiment was that the DI lesions blocked alpha conditioning. Over the 4 days of testing, response areas in the DI lesion group were small and did not increase with either paired or unpaired CS-US presentations. Successive daily means were $149,107,113$, and 89 units under the paired condition, and $101,89,51$, and 106 units under the unpaired condition (see Figure 5). In contrast, response areas in the cortical lesion group were uniformly larger than those of the DI lesion group, and revealed the presence of both pseudoconditioning and associative conditioning. Under the unpaired condition, response areas increased slightly from Day 1 to Day 2, but then declined on Days 3 and 4: successive daily means were $494,627,299$, and 420 units. Conversely, response areas under the paired condition continued to increase over Day 1 levels, and were uni- formly high on Days 2, 3, and 4: successive daily means were $577,841,881$, and 985 units.

A three-way repeated measures ANOVA (type of lesion $\times$ CS-US contiguity $\times$ days) confirmed the overall difference between the two lesion groups and the effect of CS-US contiguity in the cortical lesion group. Specifically, there was a significant effect of lesions $[F(1,12)$ $=10.92, p<.01]$, a significant lesion $\times$ day interaction $[F(3,36)=3.80, p<.05]$, and, most importantly, a significant three-way interaction between lesion type, contiguity, and days $[F(3,36)=4.82, p<.01]$. Analysis of simple interactions showed a significant interaction between CS-US contiguity and days in the cortical lesion group $[F(3,18)=8.162, p<.005]$, but not in the DI lesion group $[F(3,18)=0.47$, n.s.]. In other words, associative conditioning-defined as an increase in responding specific to contiguous presentations of CS and US - was displayed by cortically lesioned rabbits and not by DI lesioned rabbits.

\section{DISCUSSION}

The present results clearly indicate that the DI region of the cerebellum is essential for alpha and classical conditioning of eyelid responses, but not for alpha responses. 
Specifically, lesions of the DI abolished CRs to a tone and prevented alpha conditioning to a dim light, but did not impair alpha responses to either a dim light or a bright light. This pattern of deficits suggests that in the case of eyelid conditioning in the rabbit, alpha conditioning and classical conditioning share a common anatomical substrate that is distinct from the anatomical areas subserving alpha responses to bright lights. In other words, alpha conditioning to a proximal light appears to be a process by which a CR driven by a circuit through the DI becomes superimposed upon a preexisting alpha response driven by a separate pathway. This conclusion contradicts the notion that alpha conditioning and classical conditioning depend on neural changes within the alpha response pathway.

A significant contribution of the present study is the description of alpha responses to a bright proximal light, and the characterization of the conditioning when a dimmer version of the same light is paired with an airpuff US. Previous studies of eyelid conditioning in the rabbit have used a diffuse visual CS specifically to minimize the occurrence of alpha responses (e.g., Gormezano, 1972; Patterson \& Romano, 1987). The present study clearly shows that three different kinds of responses-alpha responses, CRs, and conditioned alpha responses-can be elicited in a single effector system, and that they have remarkably similar topographies. It would be interesting to determine whether this topographic similarity is due to similar operating characteristics among parallel systems or to response shaping in a final common path.

The abolition of CRs to the tone by DI lesions in the present study confirmed previous reports of the importance of this region to classically conditioned eyelid responses (McCormick \& Thompson, 1984; Yeo et al., 1985). More importantly, the CR deficits observed in every animal provided a behavioral verification of damage to the CR pathway to supplement the histological localization, thereby confirming the accurate placement of the lesions.

The principal finding of the present study was a clear dissociation between alpha responses and alpha conditioning: DI lesions impaired only alpha conditioning. After lesions of the DI, alpha responses to a bright light presented to the ipsilateral eye were increased over prelesion levels to a degree consistent with a prelesion trend toward sensitization. Responses in the control eye, contralateral to the lesion, were increased to a similar degree. In addition, initial presentations of a dim light elicited small but reliable responses from animals in both lesion groups. These results indicate that the DI lesions did not damage the alpha response pathway or produce any nonspecific sensory or motor deficits.

Despite this clear preservation of alpha responses, alpha conditioning was completely blocked by the DI lesions. Rabbits with DI lesions displayed consistently small responses over the entire training period, regardless of
CS-US contiguity. This absence of conditioning was not due to inadequate training parameters, because cortically lesioned animals developed conditioned alpha responses within a normal number of paired trials (cf. unpublished observations from Skelton et al., 1984, and Powell, Schneiderman, Elster, \& Jacobson, 1971). Instead, the absence of alpha conditioning appeared to be a specific consequence of the DI lesion, and resembled the abolition of CRs to a diffuse (non-alpha-eliciting) light CS produced by lesions of the anterior interpositus (Yeo et al., 1985). Together with the deficits in CRs to the tones, the present results indicate that the DI lesions concurrently damaged neural circuits mediating classical conditioning to the tone and alpha conditioning to the light, while sparing the circuit subserving alpha responses to the bright light.

Presumably, alpha eyelid responses could be mediated by two parallel pathways, with one passing though the DI region; alpha conditioning could be mediated by this secondary pathway. Indeed, several forms of learning are known to involve redundant pathways. Two examples are brightness discrimination in rats (Lashley, 1950) and heart rate conditioning in pigeons (Cohen, 1984). However, in the present study there was no evidence for such a pathway: DI lesions did not diminish alpha responses at all. If there was a prepotent pathway through the DI, it certainly did not make a substantial contribution to the alpha response. Therefore, there seems to be little value in distinguishing such a pathway from any other preexisting pathway subserving conditioning. After all, neuroscientists generally agree that conditioning most likely involves changes in preexisting pathways (Byrne, 1987), and calling a weakly prepotent pathway a "secondary alpha response pathway" would seem to be an unnecessary semantic distinction.

Two unexpected and possibly related observations within the present experiment suggested a role for the cerebellum in nonassociative modulations of alpha responses. First, cortical lesions appeared to enhance alpha responding in the ipsilateral eye. Second, cortical lesions may have also enhanced pseudoconditioning in the ipsilateral eye. In the absence of an unoperated control group, it is difficult to tell whether the cortical lesions facilitated nonassociative increases in alpha responses or the DI lesions blocked these increases. However, in a previous study using exactly the same dimlight CS and airpuff US, acquisition of CRs by intact rabbits did not include such early and rapid increases in response amplitudes (unpublished observations from Skelton et al., 1984). The present results provide relatively weak evidence that the cerebellar cortex normally suppresses nonassociative increases in alpha responses, in a manner reminiscent of the way that the limbic cortex suppresses behavioral responses to novel stimuli (Gabriel, Sparenborg, \& Stolar, 1987). Importantly, the present results do not suggest that the cerebellum has any role 
in the actual production of baseline alpha responses. Rather, they support the notion that neural mechanisms of associative conditioning are built upon mechanisms subserving nonassociative conditioning (cf. Kandel \& Schwartz, 1982).

In sum, the results of the present study indicate that the DI region of the cerebellum is essential to both alpha and classical conditioning of eyelid responses, but not to alpha responses themselves. By inference, if the pathways mediating alpha conditioning and classical conditioning of eyelid responses both involve the DI, and the pathway subserving unconditioned alpha responses does not, then neither form of conditioning can depend upon changes in the alpha response pathway. This conclusion contradicts the notion that alpha conditioning always involves augmentation of prepotent pathways, while supporting the related proposition that, at the neural level, alpha and classical conditioning are mediated by a common mechanism. In the case of eyelid conditioning, alpha conditioning to a light CS and classical conditioning to a tone CS seem to differ only in the magnitude of the baseline response to the CS, which may in turn depend upon the potency of separate pathways uninvolved in conditioning. In fact, eyelid conditioning to either tones or lights appears to depend on neural circuits specialized to subserve behavioral plasticity, and not on prepotent pathways that mediate preexisting reflex responses.

The present findings suggest a basic difference between conditioning of eyelid responses in rabbits and conditioning of reflex responses in reduced or simpler nervous systems. For example, conditioning of gill withdrawal in Aplysia and conditioning of leg flexion responses in acute spinal cats are both clear instances of alpha conditioning, and both are mediated by enhanced synaptic transmission in the alpha response pathway (Kandel \& Schwartz, 1982; Patterson, 1980; Patterson, Cegavske, \& Thompson, 1973). In these preparations, the neural plasticity encoding the learning resides within the reflex pathway itself. In contrast, the plasticity encoding eyelid conditioning in intact rabbits appears to be extemal to the alpha response pathway. A possible inference is that learning in intact mammalian nervous systems is mediated by anatomical systems specialized to subserve behavioral plasticity, rather than by physiological specializations within reflex pathways.

It is vital to recognize that the present results do not detract from the importance or generality of molecular studies of conditioning in reduced or simpler nervous systems. At the molecular level, the mechanisms of eyelid conditioning in rabbits and gill withdrawal conditioning in Aplysia could well be the same, even if the cellular mechanisms are different. For example, activitydependent presynaptic facilitation requires only a presynaptic convergence of CS and US information and does not require firing of the postsynaptic cell. Therefore, this mechanism should be capable of modifying synaptic transmission in pathways that are not prepotent.
However, the present findings do suggest the need for caution when trying to infer the cellular basis of conditioning in mammalian brains from studies of less complex nervous systems.

\section{REFERENCES}

Brakel, S., Babb, T., Mahnke, J., \& Verzeano, M. (1971). A compact amplifier for extracellular recording. Physiology \& Behavior, 6, 731-733.

Byrne, J. H. (1987). Cellular analysis of associative learning. Physiological Reviews, 67, 329-439.

Carew, T. J., Abrams, T. W., Hawkins, R. D., \& Kandel, E. R. (1984). The use of simple invertebrate systems to explore psychological issues related to associative learning. In D. L. Alkon \& J. Farley (Eds.), Primary neural substrates of leaming and behavioral change (pp. 169-183). New York: Cambridge University Press.

CoHEN, D. H. (1984). Identification of vertebrate neurons modified during learning: Analysis of sensory pathways. In D. L. Alkon \& J. Farley (Eds.), Primary neural substrates of leaming and behavioral change (pp. 129-154). New York: Cambridge University Press.

Gabriel, M., Sparenborg, S. P., \& Stolar, N. (1987). Hippocampal control of cingulate cortical and anterior thalamic information processing during learning in rabbits. Experimental Brain Research, 67, 131-152.

Gormezano, I. (1972). Investigations of defense and reward conditioning in the rabbit. In A. H. Black \& W. F. Prokasy (Eds.), Classical conditioning II: Current research and theory (pp. 151-181). New York: Appleton.

Gormezano, I., \& KehoE, E. J. (1975). Classical conditioning: Some methodological-conceptual issues. In W. K. Estes (Ed.), Handbook of learning and cognitive processes (Vol. 2, pp. 143-179). Hillsdale, NJ: Erlbaum.

Gormezano, I., MoORE, J. W. (1969). Classical conditioning. In M. H. Marx (Ed.), Learning: Processes (pp. 121-203). London: Collier-Macmillan.

Hull, C. L. (1934). Learning: II. The factor of the conditioned reflex. In C. Murchison (Ed.), A handbook of general experimental psychology (pp. 382-455). Worcester, MA: Clark University Press.

Kandel, E. C., \& SCHWARTZ, J. H. (1982). Molecular biology of learning: Modulation of transmitter release. Science, 218, 433-443.

Kandel, E. C., Spencer, W. A. (1968). Cellular neurophysiological approaches in the study of learning. Physiological Reviews, 48, 65-134.

LASHLEY, K. S. (1950). In search of the engram. In Symposium of the Society for Experimental Biology, 4, 454-482.

McCormick, D. A., Clark, G. A., Lavond, D. G., \& Thompson, R. F. (1982). Initial localization of the memory trace for a basic form of learning. Proceedings of the National Academy of Sciences, 79, 2731-2735.

McCormick, D., \& Thompson, R. F. (1982). A coronal atlas of the rabbit brain. Unpublished manuscript, Stanford University.

McCormick, D. A., \& Thompson, R. F. (1984). Cerebellum: Essential involvement in the classically conditioned eyelid response. Science, 223, 296-298.

Patterson, M. M. (1980). Mechanisms of classical conditioning of spinal reflexes. In R. F. Thompson, L. Hicks, \& Y. Schyrokov (Eds.), Neurophysiological mechanisms of goal-directed behavior and learning. New York: Academic Press.

Patterson, M. M., Cegavske, C. F., \& Thompson, R. F. (1973). Effects of a classical conditioning paradigm on hind-limb flexor nerve response in immobilized spinal cats. Joumal of Comparative \& Physiological Psychology, 84, 88-97.

Patterson, M. M., Romano, A. G. (1987). The rabbit in Pavlovian conditioning. In I. Gormezano, W. F. Prokasy, \& R. F. Thompson (Eds.), Classical conditioning (3rd ed., pp. 1-36). Hillsdale, NJ: Erlbaum.

Powell, D. A., Schneiderman, N., Elster, A. J., \& Jacobson, A. 
(1971). Differential classical conditioning in rabbits (Oryctolagus cuniculus) to tones and changes in illumination. Joumal of Comparative \& Physiological Psychology, 76, 267-274.

Rosenfield, M. E., Devydarts, A., \& Moore, J. W. (1985). Brachium conjunctivum and rubrobulbar tract: Brainstem projections of red nucleus essential for conditioned nictitating membrane response. Physiology \& Behavior, 34, 751-759.

Skelton, R. W., Donegan, N. H., Thompson, R. F. (1984). Superior colliculus lesions disrupt classical conditioning to visual but not auditory stimuli. Neuroscience Abstracts, 10, 132.

Solomon, P. R., Lewis, J. L., LoTurco, J. J., Steinmetz, J. E., \& Thompson, R. F. (I986). The role of the middle cerebellar pedun- cle in acquisition and retention of the rabbit's classically conditioned nictitating membrane response. Bulletin of the Psychonomic Society, 24, 75-78.

Thompson, R. F. (1986). The neurobiology of learning and memory. Science, 233, 941-947.

Yeo, C. H., Hardiman, M. J., \& Guckstein, M. (1985). Classical conditioning of the nictitating membrane response of the rabbit: $I$. Lesions of the cerebellar nuclei. Experimental Brain Research, 60, 87-98.

(Manuscript received October 30, 1987;

revision accepted for publication April 18, 1988.) 